\title{
Elucidation of the phenotypic spectrum and genetic landscape in primary and secondary microcephaly
}

\author{
Paranchai Boonsawat, MSc ${ }^{1}$, Pascal Joset, $\mathrm{PhD}^{1}$, Katharina Steindl, MD ${ }^{1}$, Beatrice Oneda, $\mathrm{PhD}^{1}$, \\ Laura Gogoll, MD¹, Silvia Azzarello-Burri, MD ${ }^{1}$, Frenny Sheth, PhD², Chaitanya Datar, MD², \\ Ishwar C. Verma, MD ${ }^{4}$, Ratna Dua Puri, MD ${ }^{4}$, Marcella Zollino, MD ${ }^{5}$, \\ Ruxandra Bachmann-Gagescu, MD ${ }^{1}$, Dunja Niedrist, MD ${ }^{1}$, Michael Papik, MSc ${ }^{1}$, \\ Joana Figueiro-Silva, PhD ${ }^{1}$, Rahim Masood, MD ${ }^{1}$, Markus Zweier, PhD ${ }^{1}$, Dennis Kraemer, MSc ${ }^{1}$, \\ Sharyn Lincoln, $\mathrm{MS}^{6}$, Lance Rodan, $\mathrm{MD}^{6,7}$, Undiagnosed Diseases Network (UDN), \\ Sandrine Passemard, $\mathrm{MD}^{8,9}$, Séverine Drunat, $\mathrm{PhD}^{9}$, Alain Verloes, $\mathrm{MD}, \mathrm{PhD}^{9}$, \\ Anselm H. C. Horn, PhD ${ }^{10}$, Heinrich Sticht, $\mathrm{PhD}^{10}$, Robert Steinfeld, MD ${ }^{11}$, Barbara Plecko, MD ${ }^{11,12}$, \\ Beatrice Latal, MD ${ }^{13}$, Oskar Jenni, MD ${ }^{13}$, Reza Asadollahi, MD, PhD ${ }^{1}$ and Anita Rauch, MD ${ }^{1,14,15}{ }^{\prime}$
}

Purpose: Microcephaly is a sign of many genetic conditions but has been rarely systematically evaluated. We therefore comprehensively studied the clinical and genetic landscape of an unselected cohort of patients with microcephaly.

Methods: We performed clinical assessment, high-resolution chromosomal microarray analysis, exome sequencing, and functional studies in 62 patients (58\% with primary microcephaly [PM], 27\% with secondary microcephaly [SM], and $15 \%$ of unknown onset).

Results: We found severity of developmental delay/intellectual disability correlating with severity of microcephaly in PM, but not SM. We detected causative variants in $48.4 \%$ of patients and found divergent inheritance and variant pattern for PM (mainly recessive and likely gene-disrupting [LGD]) versus SM (all dominant de novo and evenly LGD or missense). While centrosome-related pathways were solely identified in PM, transcriptional regulation was the most frequently affected pathway in both SM and PM.
Unexpectedly, we found causative variants in different mitochondria-related genes accounting for $\sim 5 \%$ of patients, which emphasizes their role even in syndromic PM. Additionally, we delineated novel candidate genes involved in centrosome-related pathway (SPAG5, TEDC1), Wnt signaling (VPS26A, ZNRF3), and RNA trafficking (DDX1).

Conclusion: Our findings enable improved evaluation and genetic counseling of PM and SM patients and further elucidate microcephaly pathways.

Genetics in Medicine (2019) 21:2043-2058; https://doi.org/10.1038/s41436019-0464-7

Keywords: primary microcephaly; secondary microcephaly; $\mathrm{MCPH}$; genetic counseling; mitochondria

\section{INTRODUCTION}

Microcephaly is a clinical finding defined as an occipitofrontal head circumference (OFC) of $>2$ SDs below the mean for age, sex, and ethnicity, which affects approximately $2-3 \%$ of the population worldwide. ${ }^{1}$ Individuals with microcephaly, especially those with an OFC $<-3 \mathrm{SD}$, can manifest neurological features that require medical attention and a search for the underlying etiology among environmental or, more commonly, genetic factors. ${ }^{2}$
Microcephaly is classified into primary (PM) if present at birth, and secondary (SM) if developing thereafter. ${ }^{3}$ Accordingly, PM has been shown frequently to result from early defects in neurogenesis due to abnormal regulation of mitotic division, while SM has been often linked to disruptions of later developmental processes such as myelination and synapse formation owing to abnormal endosome regulation, vesicle membrane transport, or synaptic structural support. ${ }^{4,5}$ However, neuronal migration, DNA repair, and transcription

\footnotetext{
${ }^{1}$ Institute of Medical Genetics, University of Zurich, Schlieren, Zurich, Switzerland; ${ }^{2}$ FRIGE's Institute of Human Genetics, FRIGE House, Satellite, Ahmedabad, India; ${ }^{3}$ Sahyadri Medical Genetics and Tissue Engineering Facility, Kothrud, Pune and Bharati Hospital and Research Center Dhankawadi, Pune, India; ${ }^{4}$ Institute of Medical Genetics \& Genomics, Sir Ganga Ram Hospital, Rajinder Nagar, New Delhi, India; ${ }^{5}$ Unità Operativa Complessa di Genetica Medica, Fondazione Policlinico Universitario A. Gemelli IRCCS, and Istituto di Medicina Genomica, Università Cattolica del Sacro Cuore, Rome, Italy; ${ }^{6}$ Division of Genetics and Genomics, Department of Pediatrics, Boston Children's Hospital, Boston, MA, USA; ${ }^{7}$ Department of Neurology, Boston Children's Hospital, Boston, MA, USA; ${ }^{8}$ Service de Neuropédiatrie, Hôpital Universitaire Robert Debré, APHP, Paris, France; ${ }^{9}$ Département de Génétique, Hôpital Universitaire Robert Debré, APHP, Paris, France; ${ }^{10}$ Division of Bioinformatics, Institute of Biochemistry, Friedrich-Alexander-Universität Erlangen-Nürnberg, Erlangen, Germany; ${ }^{11}$ Division of Pediatric Neurology, University Children's Hospital Zurich, Zurich, Switzerland; ${ }^{12}$ Department of Pediatrics and Adolescent Medicine, Division of General Pediatrics, Medical University of Graz, Graz, Austria; ${ }^{13}$ Child Development Center, University Children's Hospital Zurich, Zurich, Switzerland;

${ }^{14}$ Neuroscience Center Zurich, University of Zurich, Zurich, Switzerland; ${ }^{15}$ Zurich Center of Integrative Human Physiology, University of Zurich, Zurich, Switzerland.

Correspondence: Anita Rauch (anita.rauch@medgen.uzh.ch)

These authors contributed equally: Reza Asadollahi and Anita Rauch
} 
regulation-related pathways are among those affected in both PM and SM. ${ }^{4,5}$

Microcephaly can be nonsyndromic or present as an associated feature in a variety of genetic syndromes., Currently, there are over 900 OMIM phenotype entries and almost 800 genes linked to microcephaly with variable expressivity. Particularly, 18 of these genes constitute a distinct PM subclass, termed autosomal recessive primary microcephaly or microcephaly primary hereditary (MCPH), a form of microcephaly that is relatively consistent and thus far better characterized. ${ }^{6,7}$ On the other hand, SM and nonMCPH PM show considerable heterogeneity; this has not been properly studied so far and hence remained largely elusive.

Previous studies on patients with microcephaly using clinical and radiological information as well as metabolic and targeted genetic testing were able to identify causes in a small fraction of the patients $(<20 \%)$ (refs. $\left.{ }^{2,8}\right)$. Since the advent of next-generation sequencing (NGS), mainly mixed cohorts of neurodevelopmental disorders (NDDs) have been assessed where microcephalic patients accounted for $\sim 15-41 \%$ of the cases and on average $\sim 47 \%$ of them were identified with a definite cause using exome (ES) or genome sequencing (GS)..$^{9-14}$ Until now, there are only two studies that used Mendeliome sequencing or ES to evaluate known disease-causing or candidate genes in exclusive microcephaly cohorts. The first study determined a molecular diagnosis for $\sim 29 \%$ of the cases (11/38), but did not differentiate between PM and SM. ${ }^{15}$ The other study was focused on $\mathrm{PM}$ and $\mathrm{MCPH}$ from mainly consanguineous families showing the difficulties in their clinical definitions and common overlap with microcephalic primordial dwarfism, and proposed reconsideration of phenotypic boundaries. 7

Here, we performed a comprehensive genetic study on a cohort of 62 unselected clinically well-characterized patients with syndromic or nonsyndromic microcephaly of different onset using combined high-resolution chromosomal microarray analysis (CMA) and ES. Our approach sheds light on the genetic landscape of PM and SM and delineates their respective clinical and molecular characteristics. In addition to novel clinical and molecular findings in known disease genes, we identified several novel NDD/microcephaly candidate genes.

\section{MATERIALS AND METHODS \\ Patient recruitment}

Sixty-two unrelated patients, including both syndromic and nonsyndromic, were recruited from 2015 to 2017, clinically assessed in detail, and subjected to defined genetic evaluations (Figure S1). Inclusion criteria consisted of (1) an $\mathrm{OFC}>2$ SDs below the mean at birth or later, based on World Health Organization (WHO) and established growth charts; (2) no clear evidence for an acquired etiology or history of perinatal infection; and (3) without an unequivocal etiological diagnosis after clinical assessment by pediatricians and clinical geneticists (Figure S1). We performed CMA and ES for all patients, and conventional karyotyping for 45 patients including all those who remained undiagnosed after CMA and ES analysis. Genetic testing was performed as part of a research study approved by the ethics commission of the Canton of Zurich or referral centers. Written informed consent for genetic testing, publication of clinical information, and/or photographs were obtained.

\section{CMA}

CMA for evaluation of rare coding copy-number variants (CNVs) was performed on DNA extracted from peripheral blood using Affymetrix Cytoscan $\mathrm{HD}$ or cytogenetic $2.7 \mathrm{M}$ arrays as previously described. ${ }^{16}$

\section{ES and Sanger sequencing}

ES was performed on DNA extracted from peripheral blood using Agilent SureSelect XT Clinical Research Exome Kit (V5) or Human All Exon (V6) on a HiSeq 2500 System (Illumina, CA, USA) with 125-bp paired-end reads as described elsewhere. ${ }^{17}$ ES was done as trios (index patient and parents) in 58 families and duos (index patient and mother due to the lack of paternal DNA) in 4 families. ES coverage for targeted bases and off-target mitochondrial bases, and their distribution among diagnosed and undiagnosed patients, are shown in Fig. 1a. Coding plus flanking intronic $( \pm 6 \mathrm{bp})$ regions as well as 666 previously reported mitochondrial DNA variants in 37 mitochondrial genes from the MITOMAP database were analyzed using the NextGENe Software (SoftGenetics, PA, USA) (Figure S1). A second allele search for all de novo variants in recessive OMIM morbid genes or in high-level candidate genes was performed (Supplementary Materials and Methods). Selected variants from ES were confirmed by Sanger sequencing using an AB3730 capillary sequencer (Applied Biosystems, CA, USA).

\section{Variant classification}

Rare coding CNVs were classified according to Miller et al. ${ }^{18}$ Rare (minor allele frequency $[\mathrm{MAF}] \leq 2 \%$ ) sequence variants (SVs) affecting genes known to cause Mendelian disorders were classified according to the American College of Medical Genetics and Genomics (ACMG) guidelines. ${ }^{19}$ De novo, X-linked maternal, or biallelic variants affecting other genes were classified as suspected candidates, candidates, or high-level candidates according to our defined criteria (Figure S1).

\section{Functional evaluations of selected variants}

Structural modeling, cell culture, reverse transcriptase polymerase chain reaction (RT-PCR) and quantitative RTPCR (qRT-PCR), immunoblotting, immunofluorescence, and imaging were performed to evaluate functional consequences of selected variants (Supplementary Materials and Methods). 
a

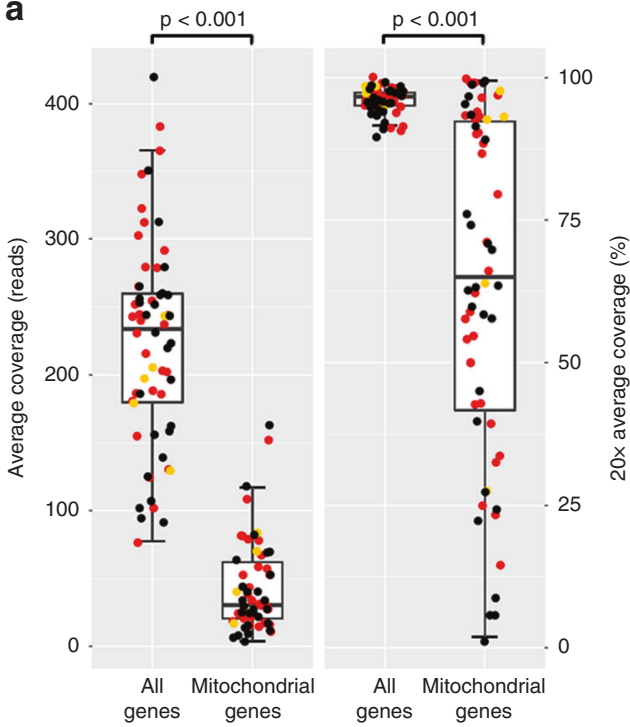

C

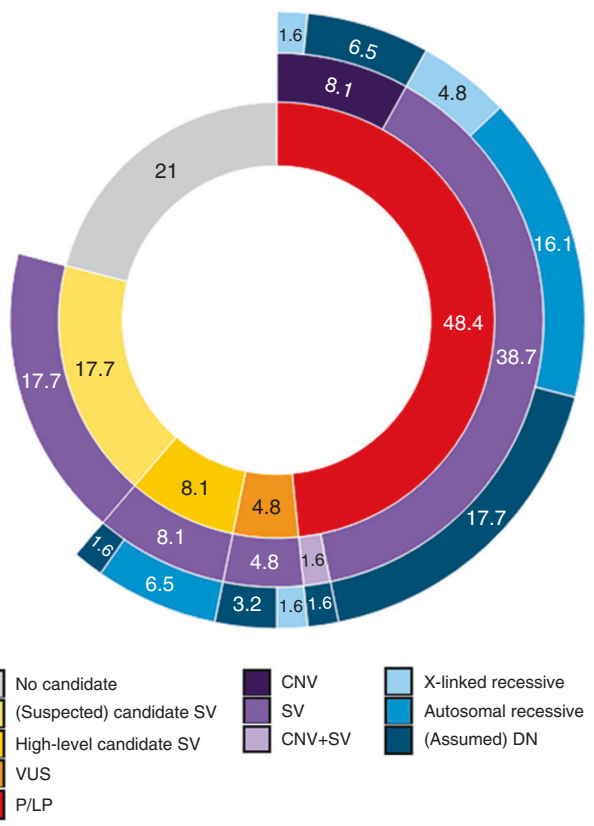

b

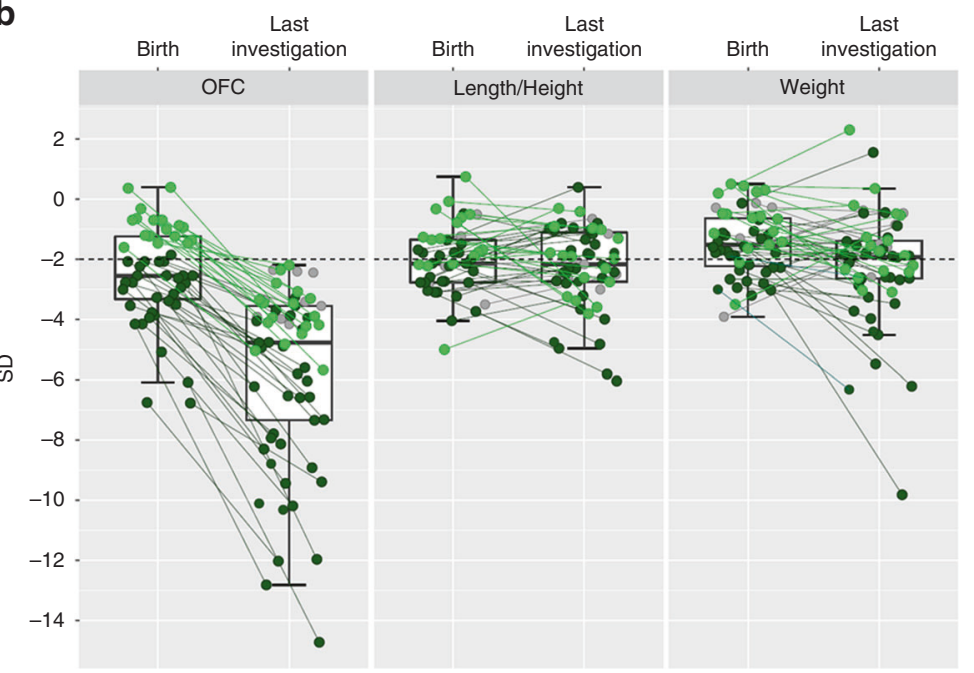

d

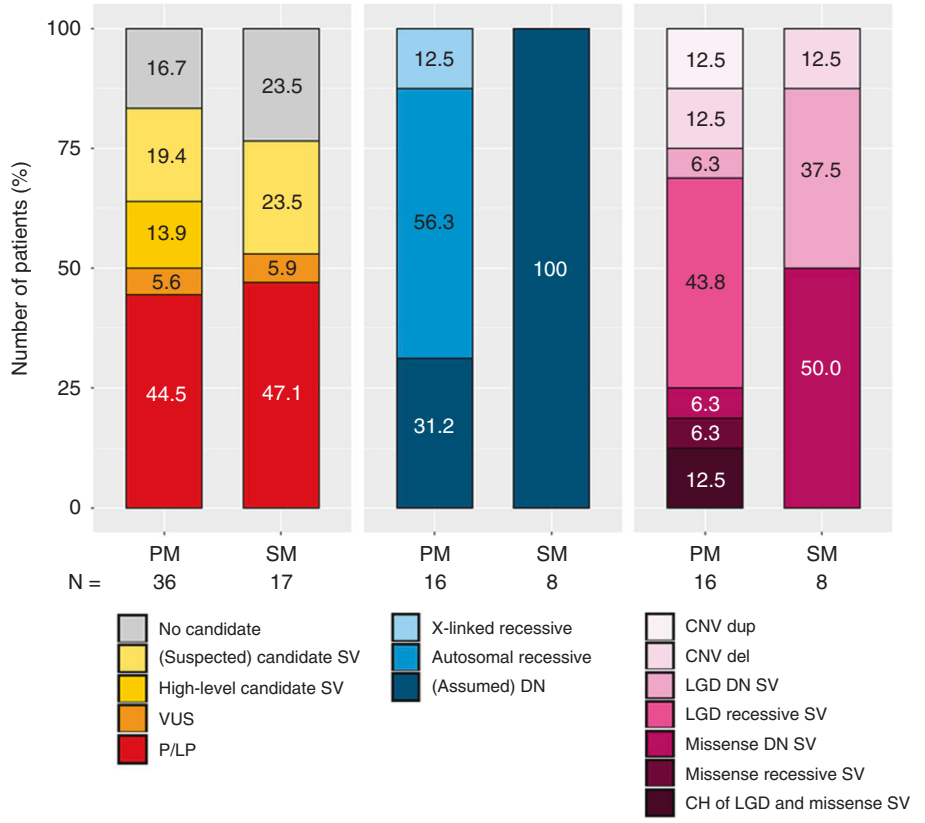

\section{RESULTS}

\section{Cohort characteristics}

We enrolled 62 unrelated patients ( 29 females, 33 males) with microcephaly of unknown etiology from 62 families (Table S1). PM and SM were determined in 36 (58.1\%) and $17(27.4 \%)$ patients, respectively (Table 1 and Fig. $1 \mathbf{b}$ ). In the other $9(14.5 \%)$ patients, the onset of microcephaly could not be determined. The median age at last investigation was 5.4 years (mean: 6.5 years, $0.8-18$ ), for PM 4.5 years (mean: 5.3 years), and for SM 4.3 years (mean: 5.7 years). The majority were of European descent (77.4\%) and the remaining were of Middle Eastern/North African (12.9\%) or Indian (9.7\%) ancestry. Nine (14.5\%) patients were born to consanguineous parents. Seven patients had one or more affected siblings. Notably, follow-up OFC measurements showed a pattern of progressive microcephaly in both PM and SM with a statistically significantly higher OFC reduction in PM than in SM patients $(p<0.001$, Wilcoxon rank-sum test) (Fig. 1b). However, $61.3 \%$ of PM and $70.6 \%$ of SM patients did not show a decline in length or height similar to that in OFC, indicating a disproportionate microcephaly in the majority of our patients (Fig. 1b). Apart from microcephaly, varying degrees of different neurological signs were reported, among which abnormal developmental milestones (developmental delay [DD] or ID) and abnormal cerebral magnetic resonance image (MRI) represented the most common associated features (Table 1). Importantly, we observed that the severity of DD/ID was significantly correlated with the severity of microcephaly among our PM patients (Figure S2A, $r=-0.43$, $p=0.01$, Spearman rank correlation with Bonferroni 
Fig. 1 Exome sequencing (ES) coverage, growth parameters, and genetic evaluations of 62 patients with microcephaly. (a) Average coverages of targeted regions (left) and 20-fold average coverages (right) of ES data for all or mitochondrial genes. On average, ES yielded an average coverage of 222fold (range: $92-419$ fold) and covered about $96 \%$ of the targeted bases with $\geq 20$ sequence reads and achieved an average off-target mitochondrial read depth of 43.6-fold (range: 3.9-163.9 fold) with a 20x average coverage of $64.4 \%$ (range: 1.9-99.4\%). Distribution of average sequencing depth and 20x coverage of the targeted region was indistinguishable among patients with P/LP variants (red dots), high-level candidate variants (yellow dots), or others (VUS, [suspected] candidate, no candidate) (black dots). Mitochondrial genes exhibited significantly lower average coverages and 20-fold average coverages (Welch $t$ test) with a higher variability in the 20-fold average coverages. PILP pathogenic or likely pathogenic, VUS variant of uncertain significance. (b) SD distributions of growth parameters measured at birth and at the time of last investigation (variable ages). Connected lines represent individual cases. SDs below -2 (dotted line) were considered microcephaly. Dark green dots: primary microcephaly (PM, 36 [58.1\%] patients); light green dots: secondary microcephaly (SM, 17 [27.4\%] patients); gray dots: unknown onset (9 [14.5\%] patients). Note that the distributions for OFC consistently show SD reductions at the last follow-up, suggesting progressiveness of microcephaly with a statistically significantly higher OFC reduction in PM compared with that in SM patients $(p<0.001$, Wilcoxon rank-sum test). However, $61.3 \%$ of PM and $70.6 \%$ of SM patients did not show a decline in length or height similar to that in OFC, indicating a disproportionate microcephaly in the majority of our patients. OFC occipitofrontal head circumference, SD standard deviation (given as standard deviation score). (c) Distribution of (potentially) relevant genetic findings in the total cohort. Inner circle shows percentages of diagnostic and uncertain findings in established disease genes, as well as likely deleterious findings in candidate genes. Middle and outer circles show the distribution of CNVs and SVs, and the inheritance pattern in the respective categories of the inner circle, respectively. P/LP variants were identified in almost $50 \%$ of the patients. Most of these variants are SVs with comparable amounts of de novo (DN) occurrence and recessive inheritance. CNV copy-number variant, SV sequence variant. (d) Genetic findings in PM and SM. Diagnostic yields between PM $(n=36)$ and SM $(n=17)$ were comparable (left panel). Predominantly recessive inheritance was identified in diagnosed PM patients ( 69\%) and dominant de novo variants in all diagnosed SM patients (middle panel). Likely gene-disrupting (LGD) variants represented the most common disease alleles ( $80 \%)$ among the diagnosed PM patients, while LGD and missense variants were equally observed among the diagnosed SM patients (right panel). CH compound heterozygous. Numbers on graphs were given as percentage.

Table 1 Summary of main clinical features in our cohort of 62 patients

Main clinical features

Number of cases

\begin{tabular}{|lc}
\hline Microcephaly & $62 / 62(100 \%)$ \\
\hline Primary & $36 / 62(58.1 \%)$ \\
\hline Secondary & $17 / 62(27.4 \%)$ \\
\hline Unknown onset & $9 / 62(14.5 \%)$ \\
\hline$D^{a}$ & $58 / 61^{b}(90.3 \%)$ \\
\hline Mild & $13 / 61(21.3 \%)$ \\
\hline Mild to moderate & $8 / 61(13.1 \%)$ \\
\hline Moderate & $12 / 61(19.7 \%)$ \\
\hline Moderate to severe & $7 / 61(11.5 \%)$ \\
\hline Severe & $15 / 61(24.6 \%)$ \\
\hline Severity not determined & $3 / 61(4.9 \%)$ \\
\hline D $^{\text {a }}$ & $24 / 28^{\mathrm{C}}(85.7 \%)$ \\
\hline Mild & $7 / 28(25 \%)$ \\
\hline Mild to moderate & $1 / 28(3.6 \%)$ \\
\hline Moderate & $8 / 28(28.6 \%)$ \\
\hline Moderate to severe & $5 / 28(17.9 \%)$ \\
\hline Severe & $3 / 28(10.7 \%)$ \\
\hline Abnormal cerebral MRI ${ }^{d}$ & $27 / 43(62.8 \%)$ \\
\hline Epilepsy/seizures & $16 / 61(26.2 \%)$ \\
\hline Ataxia or movement disorder & $15 / 61(24.6 \%)$ \\
\hline Behavioral problems & $14 / 61(23 \%)$ \\
\hline Strabismus & $6 / 61(9.8 \%)$ \\
\hline Hearing problems & $6 / 61(9.8 \%)$ \\
\hline Short stature & $20 / 61(32.8 \%)$ \\
\hline Complex congenital heart defect & $4 / 61(6.6 \%)$ \\
\hline Developen
\end{tabular}

${ }^{a}$ Developmental delay (DD) and intellectual disability (ID) were classified based on the Diagnostic and Statistical Manual of Mental Disorders (DSM-5). ${ }^{37}$

${ }^{b} 1 / 62$ cases was a fetus.

'32/61 patients were above the age of 5 years at last investigation and 28/32 were evaluated for severity of ID. 3/28 patients had learning disability and 1/28 had normal intelligence.

${ }^{d}$ Cerebral magnetic resonance image (MRI) was done for 43 patients. correction) but not among SM patients or the total cohort (Figure S2B-D). In addition, we found a significant correlation between the severity of DD/ID and abnormal cerebral MRI among the total cohort (Figure S2E, F, $p<0.01$, Fisher's exact test with Bonferroni correction).

\section{Genetic findings}

We identified pathogenic or likely pathogenic (P/LP) causative variants in $48.4 \%$ of the patients (Table 2), and variants of uncertain significance (VUS) in another $4.8 \%$ of the patients (Fig. 1c and Table S1). Furthermore, we found likely deleterious variants affecting our novel high-level candidate genes in another $8.1 \%$, affecting our novel (suspected) candidate genes in another $17.7 \%$, and we found no (candidate) causative variant in $21 \%$ of the patients (Fig. 1c). We did not find a second disease allele for any patient with inherited heterozygous likely gene-disrupting (LGD) variants in established genes known to cause recessive disorders by our alternative methods (Supplementary Materials and Methods). In six (9.7\%) patients, we found P/LP inherited heterozygous variants as secondary findings (Supplementary Results).

\section{PILP variants}

We identified pathogenic CNVs in six (9.7\%) patients (4 deletions, 2 duplications; 5 [assumed] de novo, 1 X-linked recessive inheritance) (Fig. 1c and Table 2). In one of these patients (ID74601) who had a de novo pathogenic $\sim 1.5-\mathrm{Mb}$ duplication, we identified an additional pathogenic de novo sequence variant (SV) c.3555_3556insA, p.(Ala1186Serfs ${ }^{\star} 5$ ) in KAT6A (NM_001099412.1), which likely contributes to the severity of his NDD phenotype (Table 2 and S1). Furthermore, we identified possible additional hits which may contribute to the expressivity of microcephaly in another patient (ID70688) who was identified with a pathogenic 


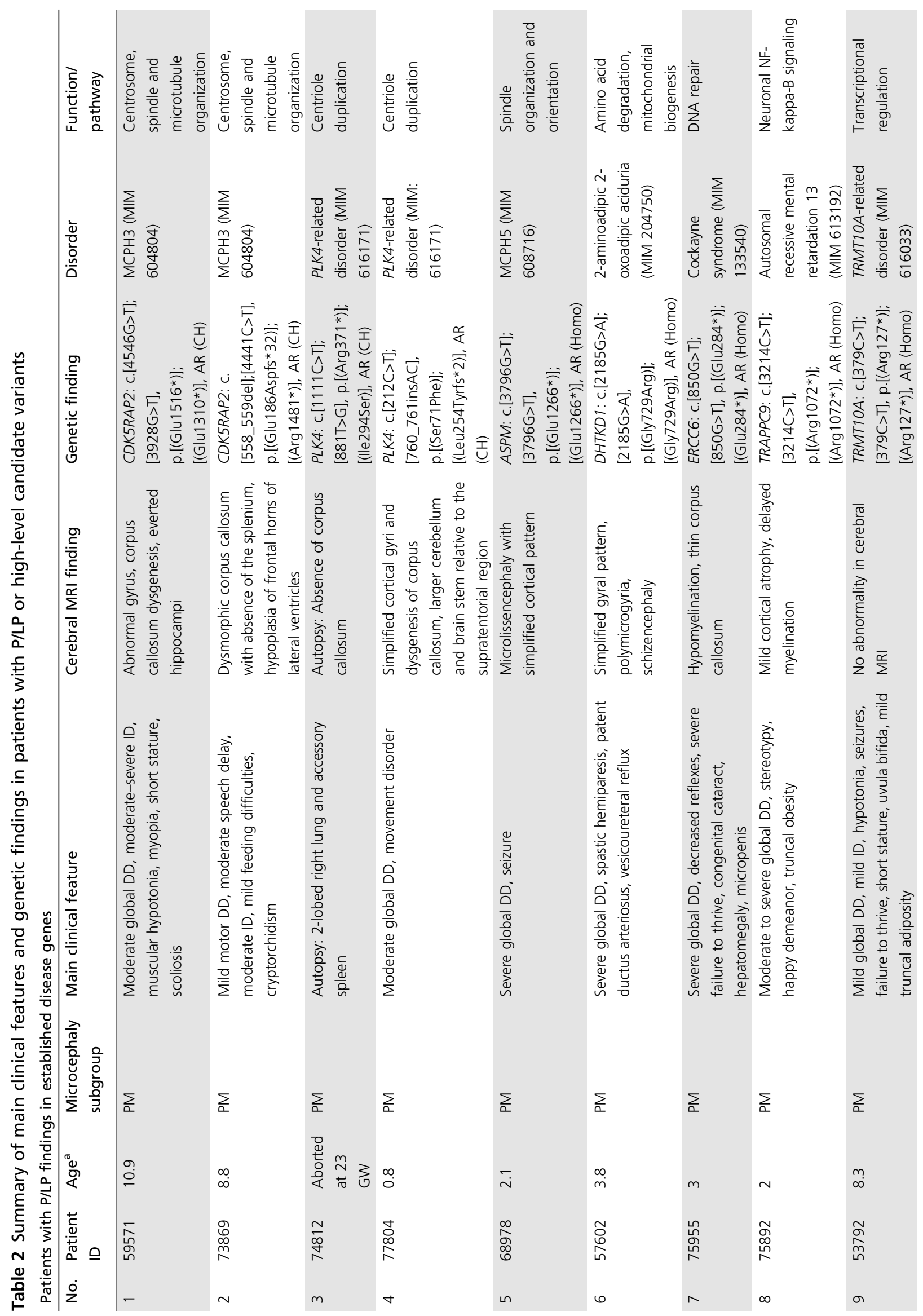




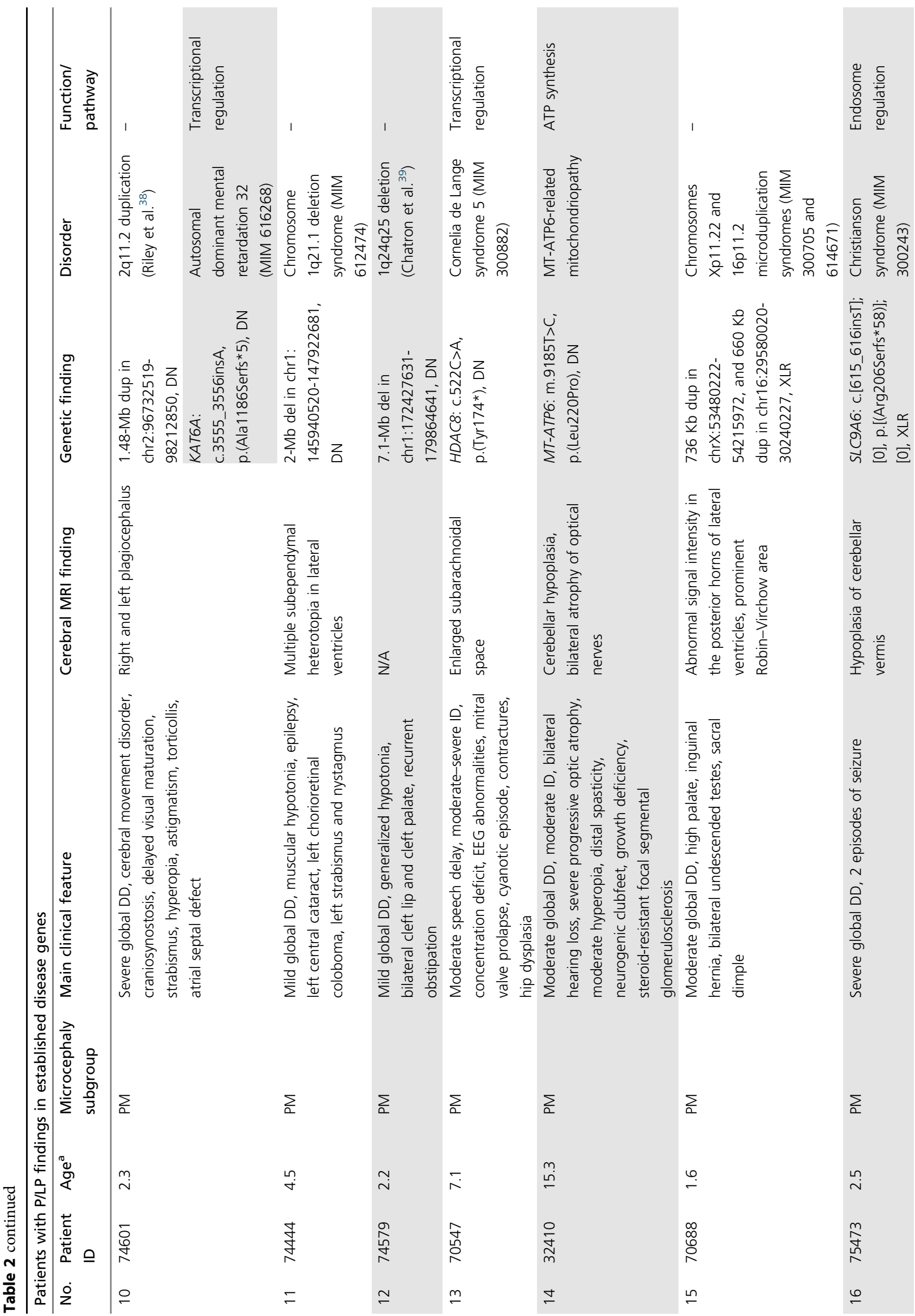




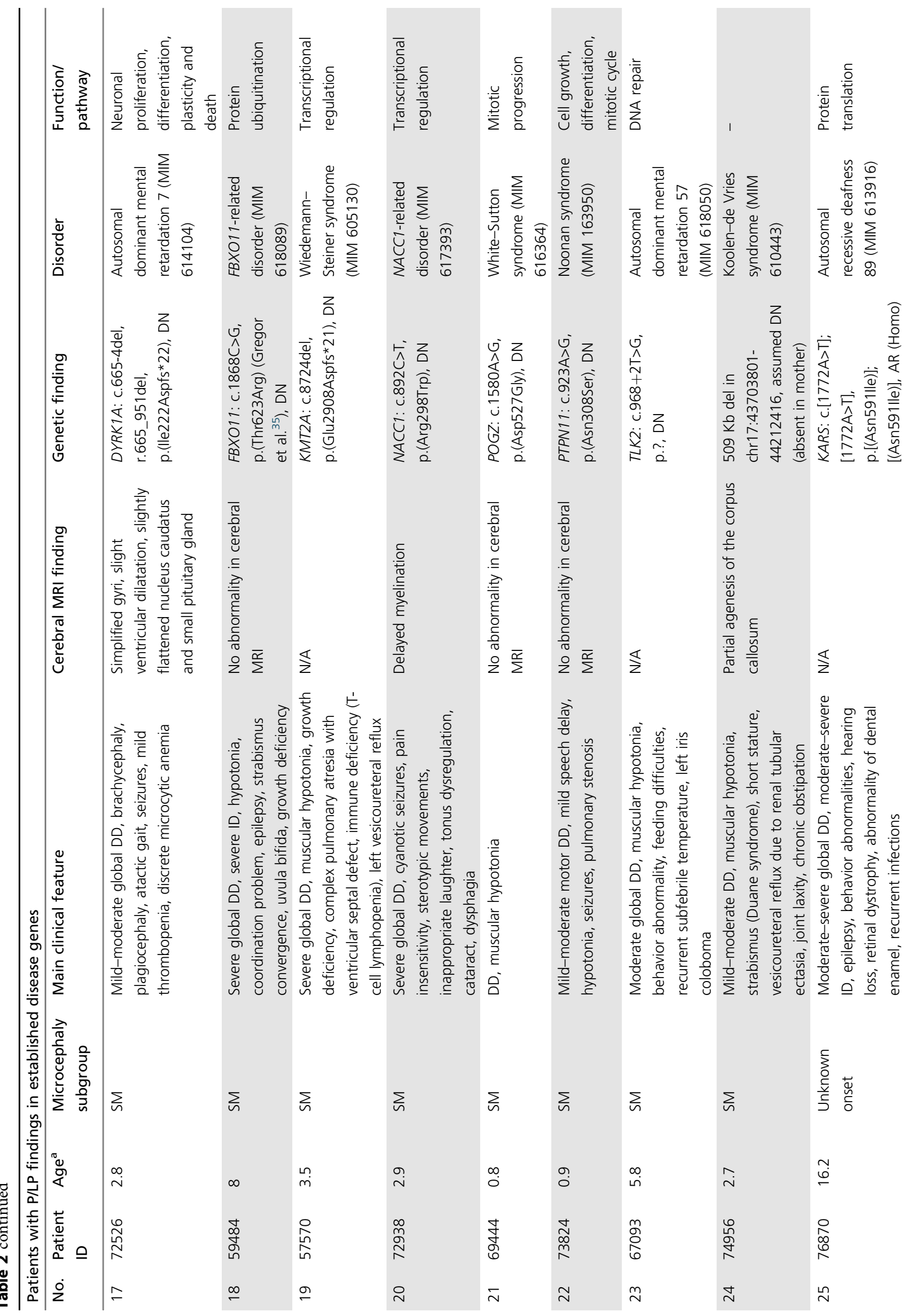



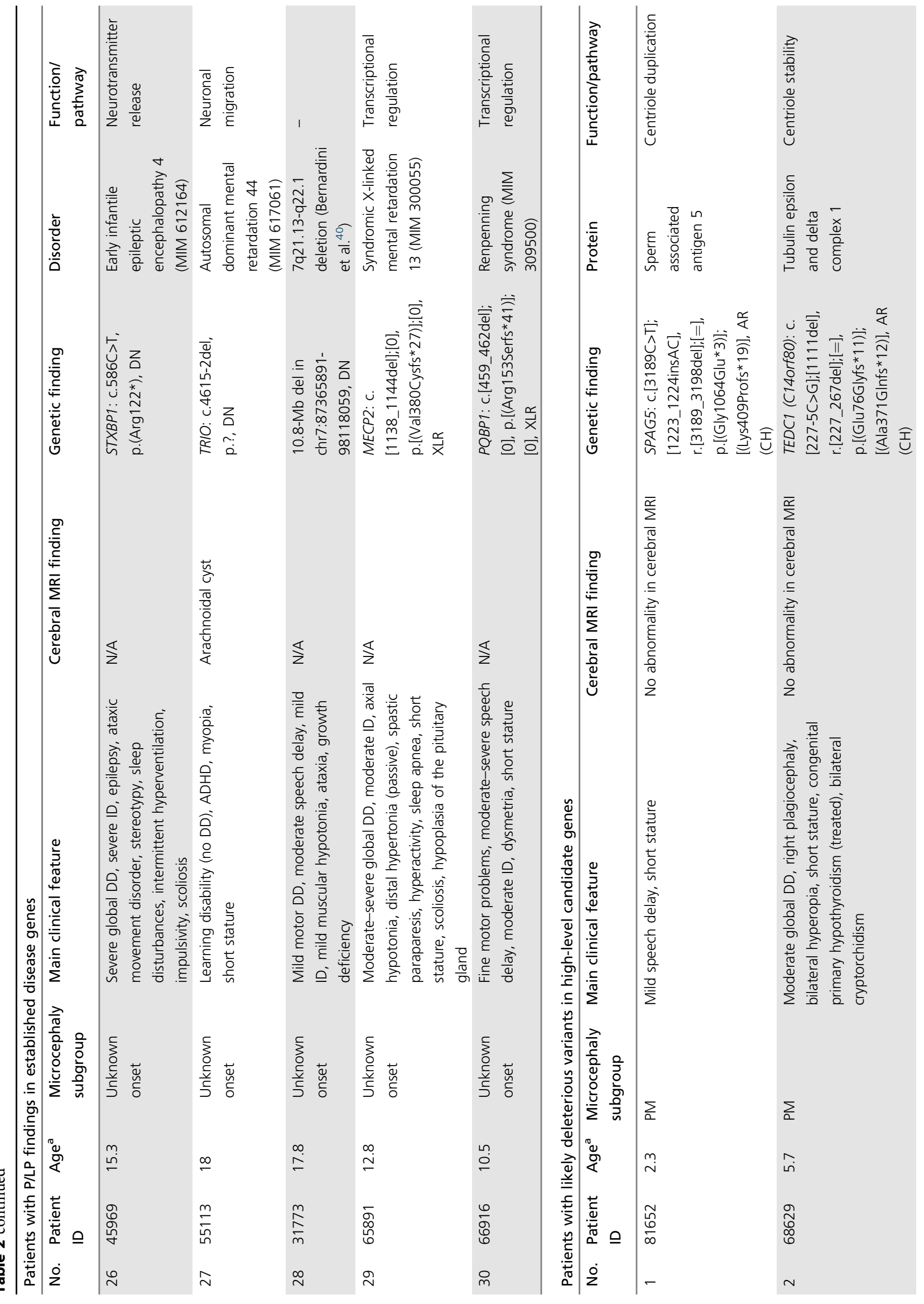
BOONSAWAT et al

ARTICLE

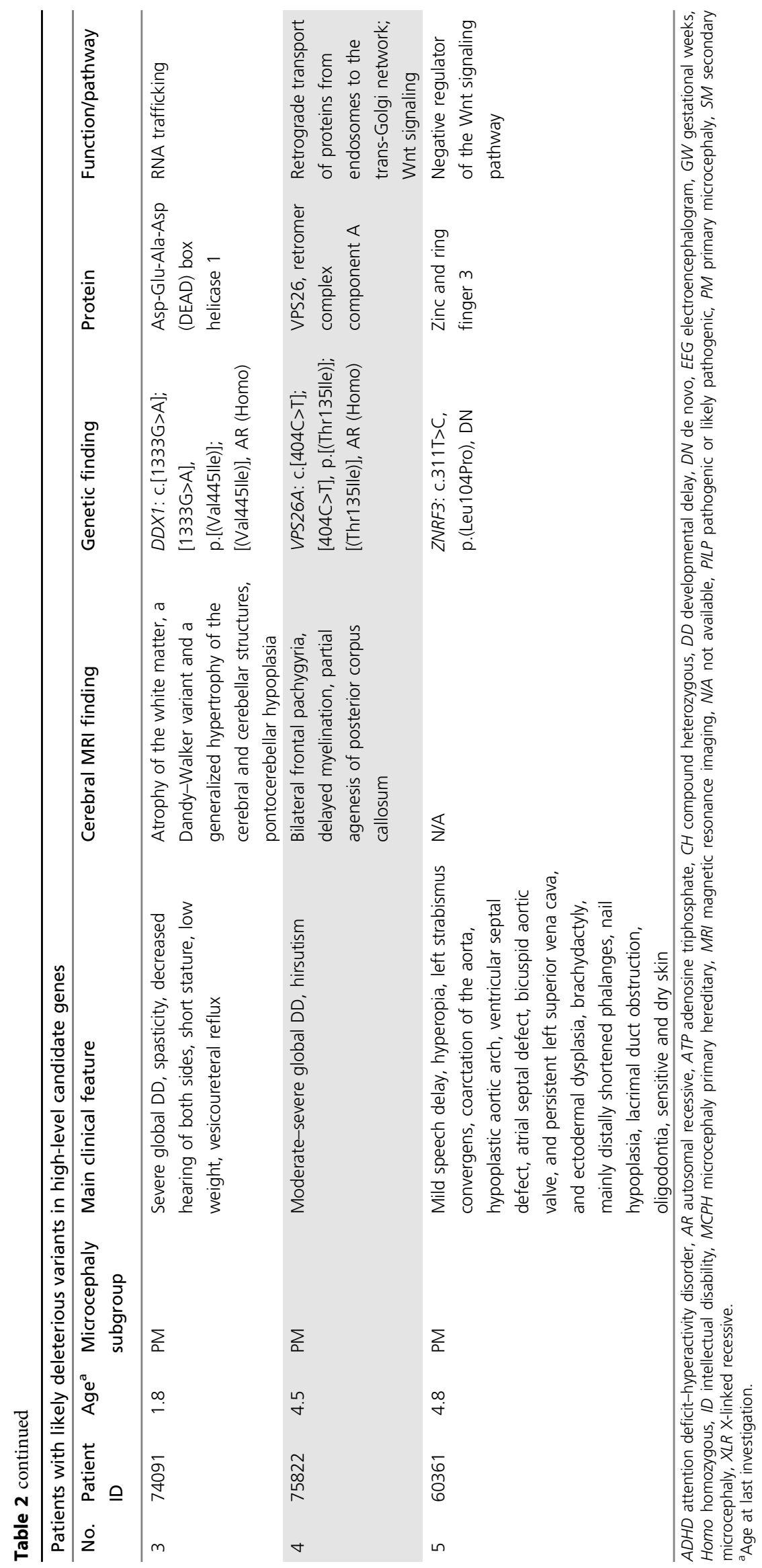

GENETICS in MEDICINE | Volume 21 | Number 9 | September 2019

2051 
Xp11.22 microduplication affecting HUWE1, PHF8, and FAM120C, a CNV known to cause X-linked ID (MIM 300705) without microcephaly (Table 2 and S1). These hits include an additional microcephaly-related $16 \mathrm{p} 11.2$ microduplication (MIM 614671) and a hemizygous nonsense unreported variant c.901C>T, p. $\left(\operatorname{Arg} 301^{\star}\right)$ in the last exon of ASB11 (NM_080873.2), which has not been yet linked to any disorder, but encodes an E3 ubiquitin protein ligase with an established role in canonical Notch signaling to regulate proper neurogenesis. ${ }^{20}$ Therefore, it is possible that these two additional variants may contribute to the manifestation of microcephaly in this patient.

Among the other 56 patients, we identified P/LP SVs affecting 22 different genes in 24 patients (CDK5RAP2 and PLK4 each in two patients), adding up to a total diagnostic yield of $48.4 \%$ (Fig. 1c). Among the diagnosed patients $(n=$ 30 ), only one ( $3 \%)$ PM patient was born to consanguineous parents. Considering the two microcephaly subclasses, we found comparable diagnostic yields of $44.5 \%$ in $\mathrm{PM}$ and $47.1 \%$ in SM (Fig. 1d). Notably, we observed recessive inheritance in $68.8 \%$ and dominant de novo variants in $31.2 \%$ of the diagnosed PM patients $(n=16)$, but dominant de novo variants in all of the diagnosed SM patients $(n=8)$. In PM, we observed mainly LGD disease alleles $(\sim 80 \%)$, while in SM, LGD and missense disease alleles were equally detected (Fig. 1d and Table 2). The affected genes in our PM subgroup belong to a variety of pathways including centrosomeassociated pathways, regulation of mitotic division, transcriptional regulation, mitochondria-related function, NF-kappa-B signaling, endosome regulation, and DNA repair, whereas the affected genes in the SM subgroup encode proteins playing roles in transcriptional regulation, cell growth and differentiation, protein ubiquitination, mitotic progression, and DNA repair (Table 2 ).

Of the P/LP SVs, seven (25\%) were recurrent variants previously reported, and 21 (75\%) were novel. Among the novel variants, we found a de novo noncanonical splice-site variant c.665-4del in DYRK1A (NM_001396.3, NG_009366.1), which was not predicted to have a splice effect, but was demonstrated by us to cause an aberrant splicing at messenger RNA (mRNA) level (exon 6 deletion, r.665_951del, p. [Ile222Aspfs ${ }^{\star} 22$ ]) (Figure S3). We also found in an aborted fetus (ID74812, Fig. 2e, f) a pathogenic nonsense PLK4 variant c. $1111 \mathrm{C}>\mathrm{T}, \mathrm{p} .\left(\operatorname{Arg} 371^{\star}\right)$ in trans with a likely pathogenic serine substitution c.881T $>$ G, p.(Ile294Ser). The latter variant, which was absent in an unaffected sibling, is the first to be located in the phosphodegron element of PLK4 and predicted to create an additional phosphorylation site likely leading to a reduced protein level via accelerated autodestruction (Table 2 and S2, Figure S4). Phenotypically, this patient presented with previously unreported organ anomalies found in autopsy (ID74812, Table 2 and S2). In an unrelated child (ID77804) with different PLK4 causative variants, we found a novel MRI finding of a large cerebellum and brain stem relative to the supratentorial region (Table 2 and S2). Other novel clinical findings in our study include uvula bifida in a patient (ID53792) with TRMT10A-related microcephaly, short stature, and impaired glucose metabolism 1 (MIM 616033), and a forgotten concept of smaller pituitary glands in Rett syndrome patients ${ }^{21}$ by our similar observation of pituitary hypoplasia in one patient (ID65891) with MECP2related X-linked mental retardation 13 (MIM 300055) (Table 2 and S1). Additionally, one patient (ID73824) was identified with a recurrent pathogenic missense variant c.923A>G, p. (Asn308Ser) in PTPN11 (NM_002834.3)_known for Noonan syndrome-and a history of perinatal asphyxia, which may contribute as an environmental factor to her microcephaly as an unusual presentation of Noonan syndrome (Table S1).

Importantly, in 1 of the 62 patients we identified a likely pathogenic variant m.9185T $>$ C, p.(Leu220Pro) in the mitochondrial gene MT-ATP6 (NC_012920.1) from ES data (ID32410, Table 2 and S1, Fig. 2a, b). This variant was observed in $59 \%$ of the reads (Figure S5A), which was also detected by a targeted panel of mitochondrial disease genes in $82 \%$ of urothelial cells (data not shown). In a phenotypically similar patient (ID76870, Table 2 and S1, Fig. 2c, d), we found a homozygous deleterious missense variant c.1772A $>$ T, p. (Asn591Ile) in a nuclear gene KARS (NM_001130089.1) which encodes a mitochondria-related protein. Our structural modeling revealed that the isoleucine substitution likely affects the protein structure and/or stability (Figure S5B). We also identified a likely pathogenic variant in another nuclear gene DHTKD1 that encodes a mitochondrial protein (Table 2 and S1). Altogether, we identified three patients with likely pathogenic variants in mitochondria-related genes, accounting for $4.8 \%$ of the total cohort.

\section{High-level candidate genes}

We identified likely deleterious variants affecting five different high-level candidate genes in five $(8.1 \%)$ patients without $\mathrm{P} /$ LP variants or VUS in established disease genes (Table 2). Four of them (SPAG5, TEDC1, VPS26A, DDX1) were affected by biallelic variants, and one (ZNFR3) by a de novo variant.

SPAG5 (sperm associated antigen 5) encodes a mitotic spindle-associated protein and has been shown to be required for regulation of mitotic spindles and recruitment of the known microcephaly gene CDK5RAP2 to the centrosome during mitosis. ${ }^{22}$ In a patient (ID81652) with PM, mild speech delay, and short stature, we found an unreported de novo frameshift variant c.1223_1224insAC, p.(Lys409Profs $\left.{ }^{\star} 19\right)$ in SPAG5 (NM_006461.3) and, by a second allele search, a maternally inherited synonymous variant c.3189C $>\mathrm{T}$, p. (Gly1063Gly) with extremely low MAF (Fig. 3a and Table S1). Sequencing of mRNA from the patient's fibroblast showed a deletion of 11 exonic bp resulting in a predicted premature stop codon (r.3189_3198del, p.[Gly1064Glu*3]) (Fig. 3b). Cycloheximide $(\mathrm{CHX})$ rescue treatment showed that both aberrant alleles were subjected to nonsense-mediated mRNA decay (NMD) with some leakiness of the splicing effect (Fig. 3b). Consistently, qRT-PCR $(\sim 75 \pm 22 \%)$ and immunoblotting $(\sim 80 \pm 26 \%)$ revealed a significantly reduced amount of the wild-type SPAG5 at both mRNA and protein levels 

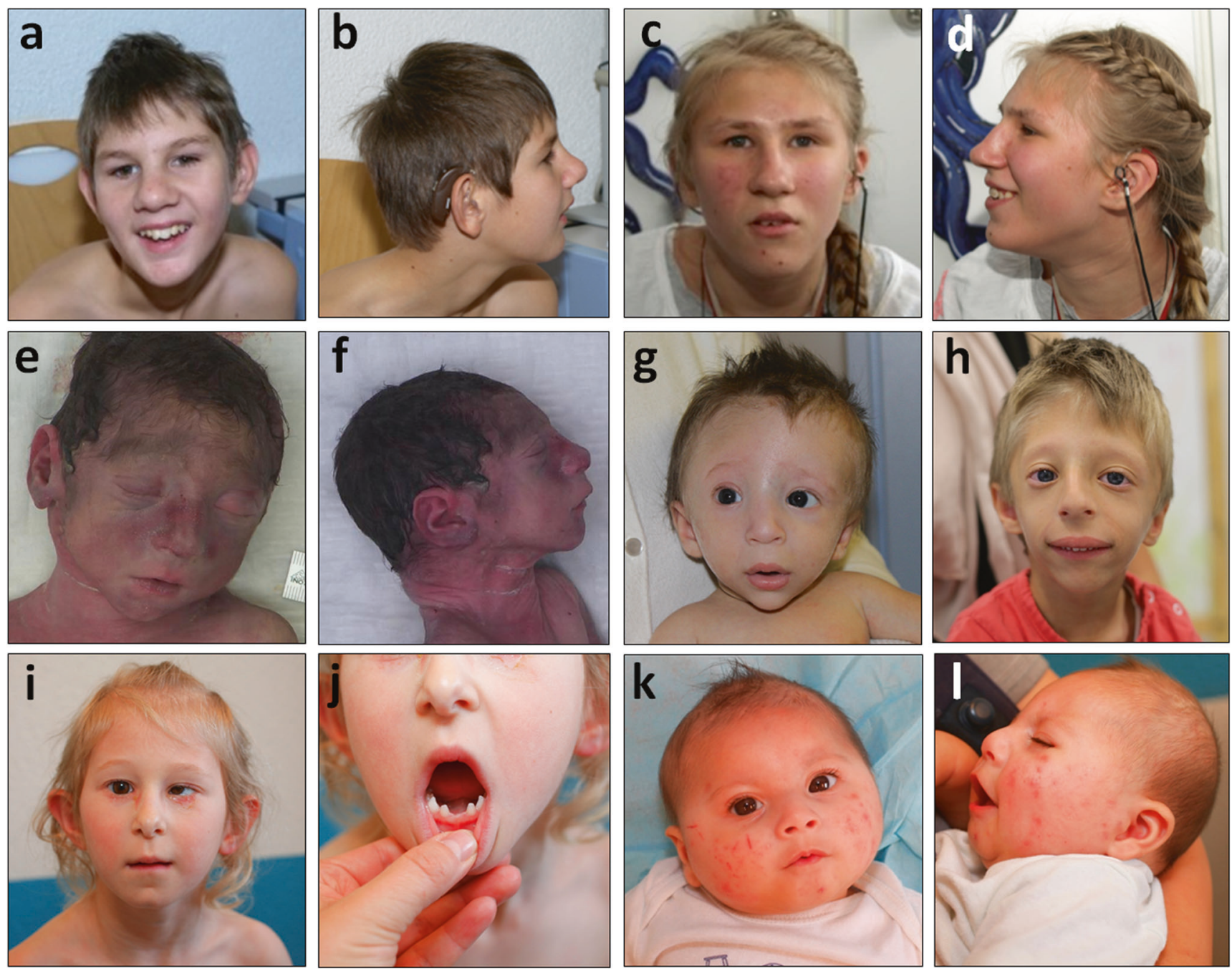

Fig. 2 Facial photographs of selected patients with expanding clinical features or harboring high-level candidate genes. (a-d) Two phenotypically similar patients (ID32410 and 76870) with likely pathogenic variants in mitochondria-related genes MT-ATP6 and KARS at 15 years 3 months and 16 years 2 months, respectively. Note apparently closely spaced eyes, long nose with bulbous tip, apparently narrow mouth with crowded teeth, and large chin. (e-f) Patient 74812 with P/LP biallelic variants in PLK4, aborted at gestational week 23. Note sloping forehead, upslanting palpebral fissures, retrognathia, and apparently large ears with increased posterior angulation. ( $\mathbf{g}-\mathbf{h})$ Patient 68629 with biallelic variants in a high-level candidate gene TEDC1 at 5 months (g) and 5 years 8 months (h). Note apparently broad forehead at young age, facial scoliosis (asymmetry with curvatures in relation to the vertical axis of the face), mild ptosis, beaked nose, apparently short ears, and micrognathia. (i-j) Patient 60361 with a de novo variant in a high-level candidate gene ZNRF3 at 4 years 9 months. Note sparse hair, left-sided microphthalmia with the secretions around both eyes due to lacrimal duct obstruction, narrow nose and nares, apparently large protruding ears, deep philtrum, thin lip vermilion (i), and oligodontia with conically shaped teeth (j). (k-I) Patient 74091 with homozygous variants in a high-level candidate gene DDX1 at 6 months. Note round face with mildly upslanting palpebral fissures, retrognathia, and apparently large ears with increased posterior angulation. P/LP pathogenic or likely pathogenic.

(Fig. 3c, d). We also observed a reduced SPAG5 intensity mainly in the centrosomal regions where it normally appears more condensed during prophase to telophase (Fig. 3e). However, morphology of the patient's fibroblasts during different cell cycle phases appeared with no obvious abnormality in the majority of cells $(>95 \%)$ (Fig. 3e), with apparently unaffected localization of the SPAG5 interacting partner CDK5RAP2 (Figure S6). Nonetheless, since we observed higher mRNA expression levels of SPAG5 in normal human induced pluripotent stem cell-derived neural progenitor cells (NPCs) compared with fibroblasts and other cell types (Fig. 3f), SPAG5 reduction may only pose deleterious effects on highly proliferative NPCs during embryonic development, which could lead to the clinical manifestations in the patient.

TEDC1 (tubulin epsilon and delta complex 1), previously known as C14ORF80, has been shown to be required for centriole stability. ${ }^{23}$ In a patient (ID68629, Fig. 2g, h and Table 2) with PM, primordial dwarfism, and moderate global $\mathrm{DD}$, we identified a noncanonical splice variant c.227-5C>G (intron 2) in trans with a frameshift variant c.1111del, p. (Ala371Glnfs*12) (last exon) in TEDC1 (NM_001134875.1) (Table 2 and Fig. $3 \mathbf{g}$ ). Sequencing of mRNA from the patient's fibroblasts showed a deletion of the first $41 \mathrm{bp}$ of exon 3 


rs113667723:
c. $918 A>\mathrm{G}$,
p.(Thr306Thr),
paternal

...TATTCTGTCCACGTGCCTGACAC...TGGTTGGCACACCAAGC. AGAGTGGCGAGCTCAT... ...GATATTCTGTCCACATGCCTGAC...CCTGGTTGGCACCAAGC. AGAGTGGTGAGCTCAT...

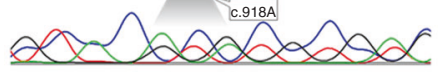

C

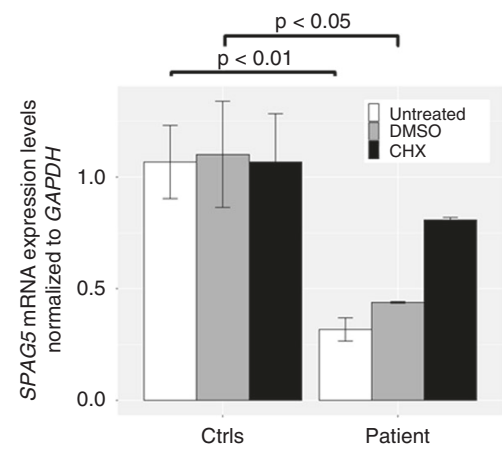

d

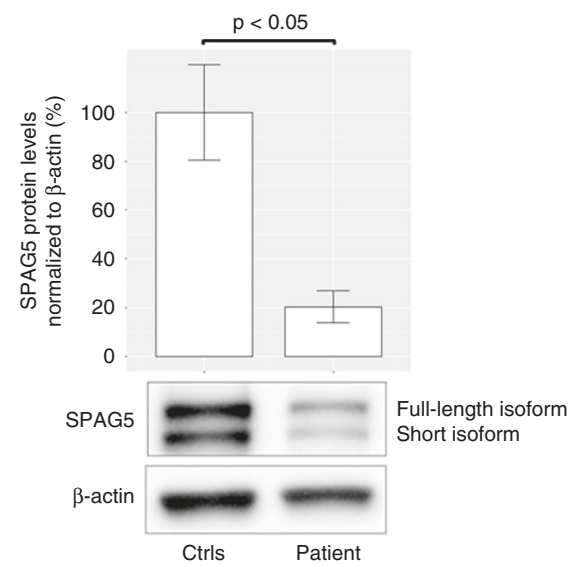

f

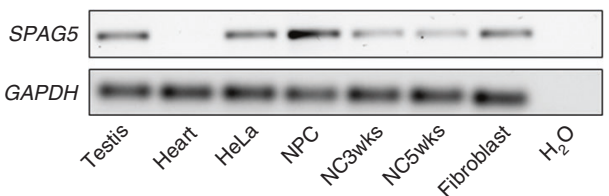

b
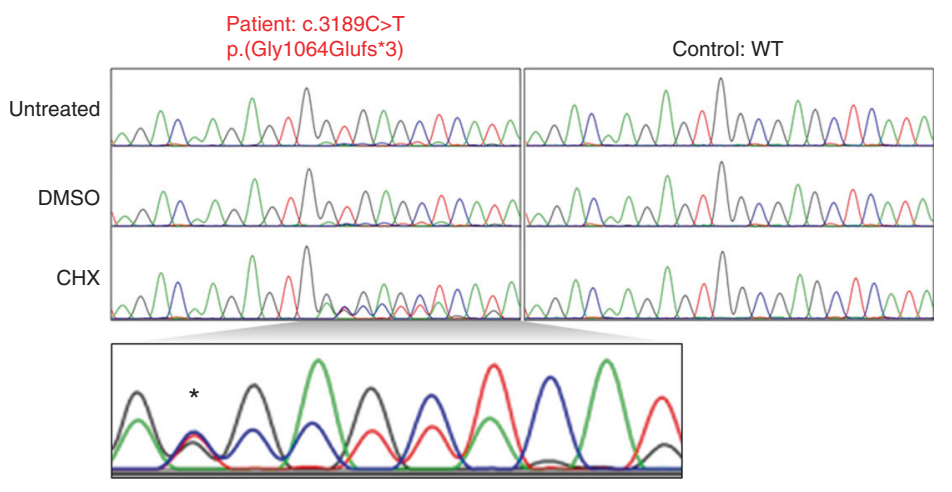

e

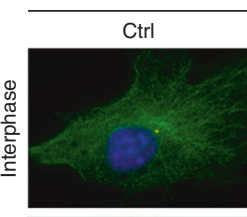

SPAG5/PCNT
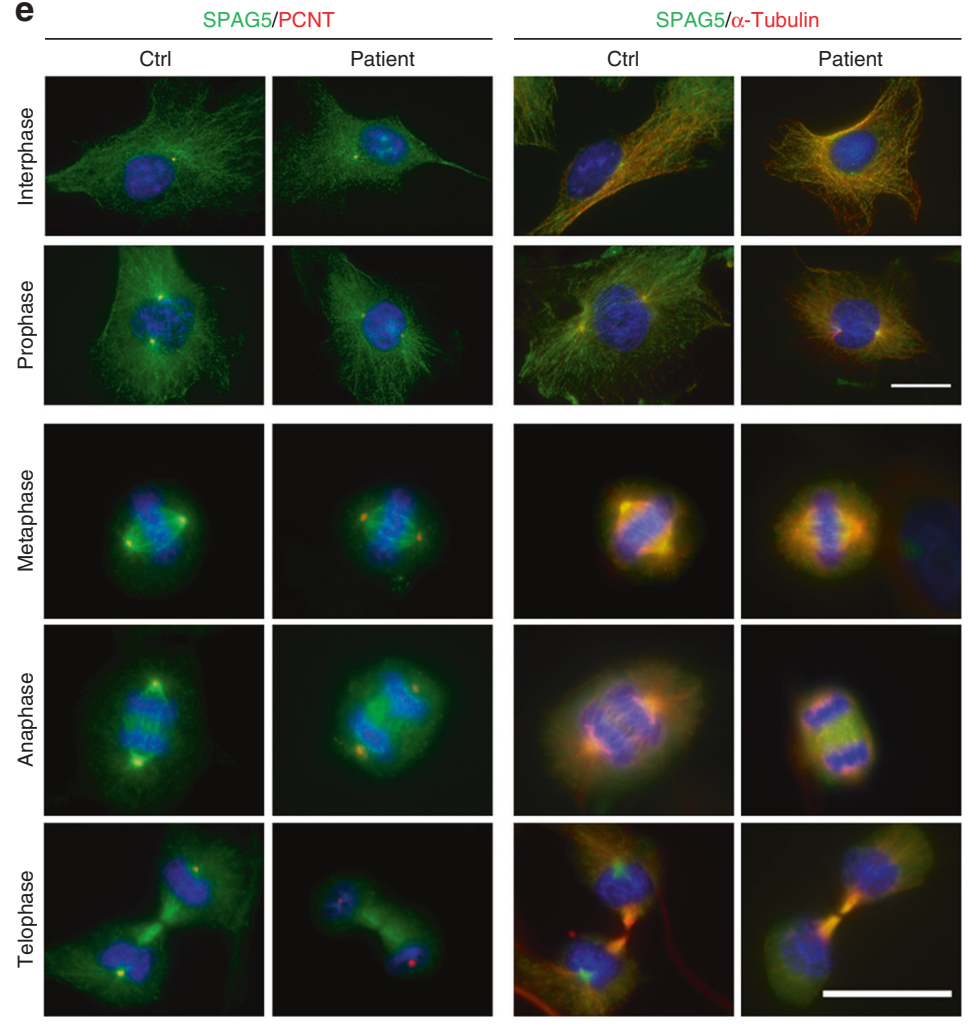

g

Exon 3 of TEDC1

..tgt(c>g)ctagAGGTCCAAGCCCGCTTGGTGAAGTCAGCACTATGCTCCCAGGGC... \begin{tabular}{ll} 
C...tgt(c) \\
c. $227-5 \mathrm{C}>\mathrm{G}$ \\
\hline
\end{tabular}

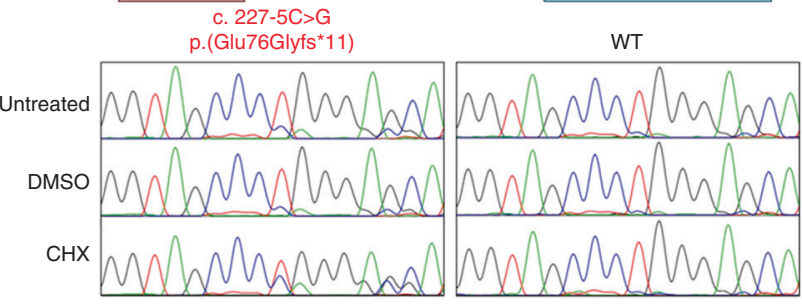

C.1111del

p.(Ala371Glnfs 12 )

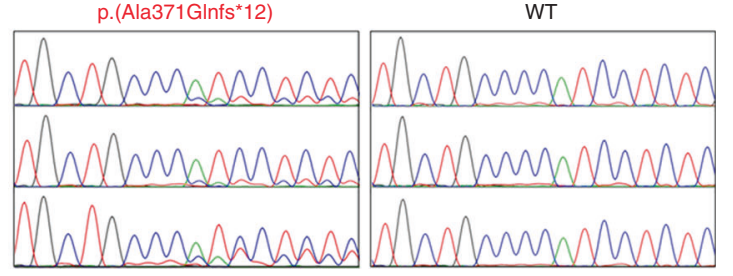


Fig. 3 Functional evaluations of high-level candidate variants in SPAG5 and TEDC1. (a) Determination of the allelic location of the de novo frameshift SPAG5 variant c.1223_1224insAC. A portion of SPAG5 sequence containing the frameshift variant and a nearby single-nucleotide polymorphism (SNP, rs113667723) was analyzed by Sanger sequencing of the patient's blood DNA, which confirmed that the frameshift SPAG5 variant was located in the paternal allele by a distinct frameshift pattern of three bases around the SNP position. Blue sequence, paternal; pink sequence, maternal; black and underlined, variants. (b) Sanger sequencing of messenger RNA (mRNA) from the patient's fibroblast (ID81652) showed a reduced amount of an aberrantly spliced transcript (due to the synonymous SPAG5 variant c.3189C >T with splice effect), which lacks the last 11 bp of exon 20, resulting in an out-of-frame mutation and a premature stop codon p.(Gly1064Glufs*3). In the magnified electropherogram of CHX, asterisk indicates rescued frameshift allele (nucleotide $\mathrm{C}$ in blue), leaky splice-site variant allele (nucleotide $\mathrm{T}$ in red), and rescued aberrantly spliced allele (nucleotide $\mathrm{G}$ in black). This means that the frameshift allele and the aberrantly spliced allele were rescued upon CHX treatment. CHX cycloheximide, DMSO dimethyl sulfoxide, WT wild type. (c) Quantitative reverse transcription polymerase chain reaction (qRT-PCR) showed significantly reduced SPAG5 mRNA levels ( 75\%) in the patient's fibroblasts (untreated and vehicle DMSO, $p<0.05$, Welch $t$ test), which were rescued upon treatment with CHX. Experiment was done in a triplicate. (d) Immunoblotting against the C-terminal terminal of SPAG5, detecting the two SPAG5 isoforms (full-length and short) and $\beta$-actin on protein extracts showed a significant reduction ( $\sim 80 \%$ ) of SPAG5 protein in the patient's fibroblasts (ID81652) ( $p<0.05$, Welch $t$ test). Note that the short isoform lacks a small portion of N-terminal of which the function has not yet been characterized. Experiment was done in a triplicate. (e) Immunostaining against SPAG5, PCNT, and $\alpha$ Tubulin shows a reduced SPAG5 intensity mainly in the centrosomal regions where it is more condensed in the control during prophase to telophase. However, morphology of the patient's fibroblasts appears with no obvious abnormality in the majority of cells (>95\%). The nuclei were visualized by DAPI staining (in blue). The scale bar represents $10 \mu \mathrm{m}$. (f) RT-PCR showed higher expression levels of SPAG5 in normal human induced pluripotent stem cell-derived neural progenitor cells (NPCs) compared with fibroblasts and other cell types including testis (positive control), heart (negative control), HeLa cell line (highly proliferative control), and NPC-derived neuronal culture at 3 (NC3wks) or 5 (NC5wks) weeks. (g) Sanger sequencing of mRNA from the patient's fibroblast (ID68629) showed a reduced amount of an aberrantly spliced transcript (due to the noncanonical splice-site TEDC1 variant C.227-5C>G that increases the activity of the cryptic splice acceptor), which lacks the first $40 \mathrm{bp}$ of exon 3, resulting in an out-of-frame mutation and a premature stop codon p.(Glu76Glyfs*11). The levels of the aberrant transcript were rescued upon CHX treatment, indicating that the aberrant transcript was subjected to nonsense-mediated decay (NMD) (see also Figure S7). On the other hand, the sequencing of the other TEDC1 variant c.1111del, which is located in the last exon, did not show a reduced amount of the aberrant transcript. Nevertheless, this variant leads to a frameshift and premature stop codon $p$. (Ala371Glnfs*12) that removes the last 50 amino acids, likely leading to a deleterious effect on the function of the TEDC1 protein, which remains to be characterized. Bar graphs show the mean \pm SEM.

(r.227_267del) predicted to result in a truncated protein (p. [Glu76Glyfs*11]) and CHX rescue treatment confirmed NMD of the aberrantly spliced transcript (Fig. $\mathbf{3 g}$ and S7). The other variant was not affected by NMD, but likely results in a Cterminally truncated protein (Fig. $\mathbf{3 g}$ and S7).

Our other high-level candidate variants, which were identified in three patients with PM and mild to severe DD, affected ZNRF3 (patient 60361, Fig. 2i, j), a negative regulator of the Wnt signaling; ${ }^{24}$ VPS26A, a mediator of Wnt transport; ${ }^{25}$ and DDX1 (patient 74091, Fig. 2k, l), a DEAD box RNA helicase, respectively (Table 2 ). Structural modeling for these missense variants predicts a variety of adverse consequences, including loss of binding affinity to the interacting protein $\mathrm{R}$-spondin for ZNRF3, loss of the ability to form a water-mediated interaction to neighboring residues for VPS26A, and steric clashes with adjacent residues for $D D X 1$, all likely affecting the protein domain stability and therefore probably contributing to the patients' clinical presentation (Table 2 and Figure S8). Notably, via GeneMatcher, ${ }^{26}$ we found an additional patient with unreported biallelic variants (c.133-8T>C, p.[?]; c.839C >T, p. [Thr280Arg]) affecting DDX1. The effects of the splice-site variant remain unknown because of no access to any other sample from this patient. However, our predictions based on the UniProt and PhosphoSitePlus databases suggest that the Thr280Arg change may cause the loss of a phosphorylation site and also interfere with posttranslational modifications of the adjacent residue Lys281, which likely affects the regulation of DDX1 interaction and/or degradation. Moreover, both patients with the recessive DDX1 variants presented with comparable neurological features including severe global DD, spastic quadriparesis, abnormal sleeping pattern, and abnormal movements/seizures, providing additional support for their pathogenicity. Nonetheless, severe microcephaly was only present in the first patient (ID74091), probably due to the contribution of possible other recessive variants in his multiple large runs of homozygosity (Table S1).

\section{Candidate and suspected candidate genes}

We found a total of 22 candidate and 26 suspected candidate genes in our cohort. Of these genes, 9 candidate $(R N F 113 A$, CEP350, SIK2, RFX7, C2CD5, KIF23, IRS2, UNC13A, PRTG) and 5 suspected candidate (NMI, LARP4B, SEC14L5, PHB2, $R A B 40 A L)$ genes were identified in $11(17.7 \%)$ patients without $\mathrm{P} / \mathrm{LP}$, VUS, or high-level candidate variants.

\section{DISCUSSION}

We have elucidated the phenotypic spectrum and genetic landscape including novel findings in PM and SM by detailed clinical assessment and combined CMA and ES of 62 unselected microcephalic patients.

In our cohort, we confirm previous findings ${ }^{2,7}$ of commonly microcephaly-associated features including DD/ID, abnormal cerebral MRI, seizures, and short stature, but in addition also frequently found movement disorders and behavioral problems. With reference to our total cohort, we corroborate previous studies showing no correlation between the degree of microcephaly and developmental performance, ${ }^{27,28}$ however, when stratifying patients for PM and SM, we unprecedentedly show here such a correlation among patients with PM. This implies that prenatal onset of OFC deceleration may pose 
stronger adverse effect on the developmental outcome. Nevertheless, our evidence of the correlation between abnormal cerebral MRI and the severity of DD/ID substantiates a previous observation of abnormal brain scans as a better reflection of developmental performance in microcephalic patients. ${ }^{29}$ Interestingly, Shaheen et al. ${ }^{7}$ observed two patterns of head growth in congenital microcephaly with severe and progressive microcephaly (pattern A) in the majority of their patients, and largely stable microcephaly (pattern B) in some patients. However, we observed pattern A, only, in PM, which might be explained by different sets of genes identified or different time points of OFC measurement. This may implicate postnatal functions of the affected genes other than only prenatal roles in proliferation of neural progenitor cells.

Etiologically, we identified P/LP variants in almost half of the cohort $(\sim 48 \%)$, accounting for a diagnostic yield that is within the higher range achieved by NGS studies on NDDs, ${ }^{9-14}$ but is more than three times that of the previous study evaluating 680 microcephalic children (15\%) using nonNGS methods, ${ }^{2}$ further supporting the effectiveness of ES for routine diagnostic testing. In addition, we have identified VUS and candidate variants in $\sim 31 \%$ of the patients. Therefore, our diagnostic yield will likely increase over time as further supporting evidence for the affected genes becomes available.

We also highlighted the importance of evaluating relevant noncanonical splice-site variants through our examples of a synonymous exonic variant in SPAG5, and a -4 intronic variant in DYRK1A, both of which caused aberrant splicing and subsequent NMD. Therefore, it is crucial to investigate such variants, and to validate those with benign in silico predictions that might be false negative due to the complexity of splicing control.

Previously, inborn errors of metabolism including mitochondriopathies have been identified in 3\% of microcephalic patients. ${ }^{2}$ However, the specific percentage of molecularly diagnosed mitochondrial disorders in microcephalic patients has not been reported so far. Our identification of LP variants in mitochondrial and mitochondria-related nuclear genes in $\sim 5 \%$ of the patients highlights the significance of mitochondrial disorders even in PM where mitochondriopathies may have been underdiagnosed. Notwithstanding, due to the mitochondrial heteroplasmy and highly variable coverage of mitochondrial genes in ES data (Fig. 1a), ${ }^{30}$ a targeted assessment of the mitochondrial DNA should be considered.

Despite the comparable diagnostic yields between PM $(\sim 44 \%)$ and SM $(\sim 47 \%)$ in our cohort, we illustrate different predominant modes of inheritance and types of causative variants between them. Our observation of predominantly recessive inheritance and biallelic LGD variants in PM patients suggests that complete protein absence may represent the most common cause of PM, which is in line with the findings in $\mathrm{MCPH}$ genes. ${ }^{6}$ On the other hand, dominant de novo LGD or assumed loss-of-function (LoF) missense variants, which we frequently observed in SM patients, suggest haploinsufficiency as a frequent pathomechanism in SM. This difference in inheritance pattern is not explained by a consanguinity bias in diagnosed PM patients, since only 1 of 16 diagnosed PM patients is an offspring of consanguineous parents. Consistent with previous studies, ${ }^{4,5}$ disease-causing genes identified in our cohort also encode proteins of various pathways, among which transcriptional regulation and DNA damage response are the most frequent in both PM and SM. However, centrosome-associated pathways are exclusively implicated in PM with autosomal recessive inheritance, which highlights their crucial function in cell division during neurogenesis. ${ }^{4,5}$ Notably, we observed the progressiveness of microcephaly not only in SM patients, but also in all our PM patients, which implicates postnatal defects in neural maintenance and synaptogenesis in both microcephaly subgroups.

Within the undiagnosed patients, we were able to identify five high-level candidate genes, all in patients with PM. Of these five genes, two (SPAG5 and TEDC1) encode centrosomal proteins, two (ZNRF3 and VPS26A) Wnt signalingrelated proteins, ${ }^{24,25}$ and one (DDX1) an RNA trafficking protein. ${ }^{31}$ In addition to the known centrosomal functions in regulating neuronal progenitor proliferation, ${ }^{32} \mathrm{Wnt}$ signaling has been shown to be essential for transition between symmetrical and nonsymmetrical cell division in human neural stem cells, ${ }^{33}$ and RNA trafficking to be involved in mRNA translation control of proteins that regulate the balance between maintenance and differentiation of radial glial progenitors and thereby development of the embryonic cortex. ${ }^{31}$ Therefore, compromise in the function of these proteins may in fact lead to defects in neurogenesis and hence primary microcephaly. However, we suggest considering all our candidate genes for NDD in general, due to the variable presentation of microcephaly in non-MCPH patients. ${ }^{34,35}$ This variability has been recently demonstrated for FBXO11related NDD, in which fewer than $25 \%$ of the patients presented with microcephaly. ${ }^{35}$

Clinical variability is often observed in NDDs, even in those with established causative genes, which has been, in some instances, attributed to additional genetic factors. ${ }^{36}$ In our cohort, we were able to identify additional genetic hits or a perinatal event likely contributing to the severity of ID or the presence of microcephaly in three patients. However, individualized explanation for all variable NDD presentations will require a comprehensive understanding of an individual's genetic as well as epigenetic status.

In conclusion, we showed that microcephaly is highly heterogeneous both phenotypically and genetically. By using a combined high-resolution CNV and ES analyses, we achieved an effective diagnostic yield of $\sim 48 \%$ and in addition proposed five novel NDD/microcephaly candidate genes with supporting evidence. We also shed some light on distinct as well as common characteristics of the two microcephaly subclasses PM and SM, which helps with better management of the patients and understanding of the underlying pathways involved in human brain development. 


\section{SUPPLEMENTARY INFORMATION}

The online version of this article (https://doi.org/10.1038/s41436019-0464-7) contains supplementary material, which is available to authorized users.

\section{ACKNOWLEDGEMENTS}

We sincerely thank the affected individuals and their families for participation and their permission to publish the results. This research was supported by ERA-NET grant "Euromicro" (SNF 31ER30_154238 to AR) and radiz-Rare Disease Initiative Zurich, clinical research priority program, University of Zurich. An additional patient with DDX1 biallelic variants reported in this manuscript was found via collaboration with the Undiagnosed Diseases Network (UDN) supported by the National Institutes of Health (NIH) Common Fund, through the Office of Strategic Coordination/Office of the NIH Director under award number U01HG007690. The content of this manuscript is solely the responsibility of the authors and does not necessarily represent the official views of the National Institutes of Health.

\section{DISCLOSURE}

The authors declare no conflicts of interest.

Publisher's note: Springer Nature remains neutral with regard to jurisdictional claims in published maps and institutional affiliations.

\section{REFERENCES}

1. von der Hagen M. Diagnostic approach to primary microcephaly. Neuropediatrics. 2017;48:133-134.

2. von der Hagen M, Pivarcsi M, Liebe J, et al. Diagnostic approach to microcephaly in childhood: a two-center study and review of the literature. Dev Med Child Neurol. 2014;56:732-741.

3. Woods CG, Parker A. Investigating microcephaly. Arch Dis Child. 2013:98:707-713.

4. Alcantara D, O'Driscoll M. Congenital microcephaly. Am J Med Genet C Semin Med Genet. 2014;166C:124-139.

5. Seltzer LE, Paciorkowski AR. Genetic disorders associated with postnatal microcephaly. Am J Med Genet C Semin Med Genet. 2014;1 66C:140-155.

6. Jayaraman $\mathrm{D}, \mathrm{Bae} \mathrm{BI}$, Walsh CA. The genetics of primary microcephaly. Annu Rev Genomics Hum Genet. 2018;19:177-200.

7. Shaheen R, Maddirevula S, Ewida N, et al. Genomic and phenotypic delineation of congenital microcephaly. Genet Med. 2018 Sep 14; https://www.nature.com/articles/s41436-018-0140-3.

8. Dahlgren L, Wilson RD. Prenatally diagnosed microcephaly: a review of etiologies. Fetal Diagn Ther. 2001;16:323-326.

9. Hamdan FF, Srour M, Capo-Chichi JM, et al. De novo mutations in moderate or severe intellectual disability. PLoS Genet. 2014;10: e1004772.

10. Najmabadi $H, H u H$, Garshasbi $M$, et al. Deep sequencing reveals 50 novel genes for recessive cognitive disorders. Nature. 2011;478:57-63.

11. Need AC, Shashi V, Hitomi $Y$, et al. Clinical application of exome sequencing in undiagnosed genetic conditions. I Med Genet. 2012;49:353-361.

12. Gilissen C, Hehir-Kwa JY, Thung DT, et al. Genome sequencing identifies major causes of severe intellectual disability. Nature. 2014;511:344-347.

13. Alazami AM, Patel N, Shamseldin HE, et al. Accelerating novel candidate gene discovery in neurogenetic disorders via whole-exome sequencing of prescreened multiplex consanguineous families. Cell Rep. 2015;10: 148-161.

14. Thevenon J, Duffourd Y, Masurel-Paulet A, et al. Diagnostic odyssey in severe neurodevelopmental disorders: toward clinical whole-exome sequencing as a first-line diagnostic test. Clin Genet. 2016;89: 700-707.

15. Rump $\mathrm{P}$, Jazayeri $\mathrm{O}$, van Dijk-Bos $\mathrm{KK}$, et al. Whole-exome sequencing is a powerful approach for establishing the etiological diagnosis in patients with intellectual disability and microcephaly. BMC Med Genomics. 2016;9:7.

16. Asadollahi $R$, Oneda $B$, Joset $P$, et al. The clinical significance of small copy number variants in neurodevelopmental disorders. J Med Genet. 2014;51:677-688.

17. Plecko $B$, Zweier $M$, Begemann $A$, et al. Confirmation of mutations in PROSC as a novel cause of vitamin B 6-dependent epilepsy. J Med Genet. 2017;54:809-814.

18. Miller DT, Adam MP, Aradhya S, et al. Consensus statement: chromosomal microarray is a first-tier clinical diagnostic test for individuals with developmental disabilities or congenital anomalies. Am J Hum Genet. 2010;86:749-764.

19. Richards S, Aziz N, Bale S, et al. Standards and guidelines for the interpretation of sequence variants: a joint consensus recommendation of the American College of Medical Genetics and Genomics and the Association for Molecular Pathology. Genet Med. 2015;17:405-424.

20. Sartori da Silva MA, Tee JM, Paridaen J, et al. Essential role for the dAsb11 cul5 Box domain for proper notch signaling and neural cell fate decisions in vivo. PLoS One. 2010;5:e14023.

21. Murakami JW, Courchesne E, Haas RH, Press GA, Yeung-Courchesne R. Cerebellar and cerebral abnormalities in Rett syndrome: a quantitative MR analysis. AJR Am J Roentgenol. 1992;159:177-183.

22. Kodani A, Yu TW, Johnson JR, et al. Centriolar satellites assemble centrosomal microcephaly proteins to recruit CDK2 and promote centriole duplication. elife. 2015;4:e07519.

23. Breslow DK, Hoogendoorn S, Kopp AR, et al. A CRISPR-based screen for Hedgehog signaling provides insights into ciliary function and ciliopathies. Nat Genet. 2018;50:460-471.

24. Hao HX, Xie $Y$, Zhang $Y$, et al. ZNRF3 promotes Wnt receptor turnover in an R-spondin-sensitive manner. Nature. 2012;485:195-200.

25. Yang PT, Lorenowicz MJ, Silhankova M, Coudreuse DY, Betist MC, Korswagen HC. Wnt signaling requires retromer-dependent recycling of MIG-14/Wntless in Wnt-producing cells. Dev Cell. 2008;14: 140-147.

26. Sobreira N, Schiettecatte F, Valle D, Hamosh A. GeneMatcher: a matching tool for connecting investigators with an interest in the same gene. Hum Mutat. 2015;36:928-930.

27. Bolduc FV, Shevell MI. Corrected head circumference centiles as a possible predictor of developmental performance in high-risk neonatal intensive care unit survivors. Dev Med Child Neurol. 2005;47:766-770.

28. Baxter PS, Rigby AS, Rotsaert MH, Wright I. Acquired microcephaly: causes, patterns, motor and IQ effects, and associated growth changes. Pediatrics. 2009;124:590-595.

29. Custer DA, Vezina LG, Vaught DR, et al. Neurodevelopmental and neuroimaging correlates in nonsyndromal microcephalic children. J Dev Behav Pediatr. 2000;21:12-18.

30. Picardi E, Pesole G. Mitochondrial genomes gleaned from human wholeexome sequencing. Nat Methods. 2012;9:523-524.

31. Vessey JP, Amadei G, Burns SE, Kiebler MA, Kaplan DR, Miller FD. An asymmetrically localized Staufen2-dependent RNA complex regulates maintenance of mammalian neural stem cells. Cell Stem Cell. 2012; 11:517-528.

32. Hu WF, Chahrour MH, Walsh CA. The diverse genetic landscape of neurodevelopmental disorders. Annu Rev Genomics Hum Genet. 2014;15:195-213.

33. Bengoa-Vergniory N, Gorrono-Etxebarria I, Gonzalez-Salazar I, Kypta RM. A switch from canonical to noncanonical Wnt signaling mediates early differentiation of human neural stem cells. Stem Cells. 2014; 32:3196-3208.

34. Laugel V, Dalloz C, Durand M, et al. Mutation update for the CSB/ERCC6 and CSA/ERCC8 genes involved in Cockayne syndrome. Hum Mutat. 2010;31:113-126.

35. Gregor A, Sadleir LG, Asadollahi R, et al. De novo variants in the F-box protein $\mathrm{FBXO11}$ in 20 individuals with a variable neurodevelopmental disorder. Am J Hum Genet. 2018;103:305-316.

36. Pizzo $L$, Jensen $M$, Polyak $A$, et al. Rare variants in the genetic background modulate cognitive and developmental phenotypes in individuals carrying disease-associated variants. Genet Med. 2018 
Sep 7; https://www.nature.com/articles/s41436-018-0266-3 [Epub ahead of print].

37. American Psychiatric Association. Diagnostic and statistical manual of mental disorders, fifth edition (DSM-5). American Psychiatric Association Publishing, Arlington, VA. 2013.

38. Riley KN, Catalano LM, Bernat JA, et al. Recurrent deletions and duplications of chromosome $2 q 11.2$ and $2 q 13$ are associated with variable outcomes. Am J Med Genet A. 2015;167A:26642673.

39. Chatron N, Haddad V, Andrieux J, et al. Refinement of genotypephenotype correlation in 18 patients carrying a 1q24q25 deletion. Am J Med Genet A. 2015:167A:1008-1017.

40. Bernardini L, Palka C, Ceccarini $C$, et al. Complex rearrangement of chromosomes 7q21.13-q22.1 confirms the ectrodactyly-deafness locus and suggests new candidate genes. Am J Med Genet A. 2008;1 46A:238-244.
Open Access This article is licensed under a Creative Commons (c) (i) (3) $\Theta$ Attribution-NonCommercial-NoDerivatives 4.0 International License, which permits any non-commercial use, sharing, distribution and reproduction in any medium or format, as long as you give appropriate credit to the original author(s) and the source, and provide a link to the Creative Commons license. You do not have permission under this license to share adapted material derived from this article or parts of it. The images or other third party material in this article are included in the article's Creative Commons license, unless indicated otherwise in a credit line to the material. If material is not included in the article's Creative Commons license and your intended use is not permitted by statutory regulation or exceeds the permitted use, you will need to obtain permission directly from the copyright holder. To view a copy of this license, visit http://creativecommons.org/licenses/by-nc-nd/4.0/.

(C) The Author(s) 2019 